\title{
Instability of thermocapillary-buoyancy convection in droplet migration
}

Cite as: Phys. Fluids 31, 122101 (2019); https://doi.org/10.1063/1.5125846

Submitted: 30 August 2019 . Accepted: 07 November 2019 . Published Online: 02 December 2019

Kai-Xin Hu (D), Chen-Yi Yan, and Qi-Sheng Chen
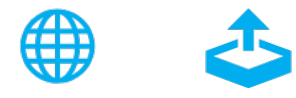

View Online

Export Citation

\section{ARTICLES YOU MAY BE INTERESTED IN}

An interface-compressed diffuse interface method and its application for multiphase flows Physics of Fluids 31, 122102 (2019); https://doi.org/10.1063/1.5116035

Instabilities of particle-laden layers in the stably stratified environment

Physics of Fluids 31, 124101 (2019); https://doi.org/10.1063/1.5123317

Rapid mixing in microchannel using standing bulk acoustic waves

Physics of Fluids 31, 122001 (2019); https://doi.org/10.1063/1.5126259

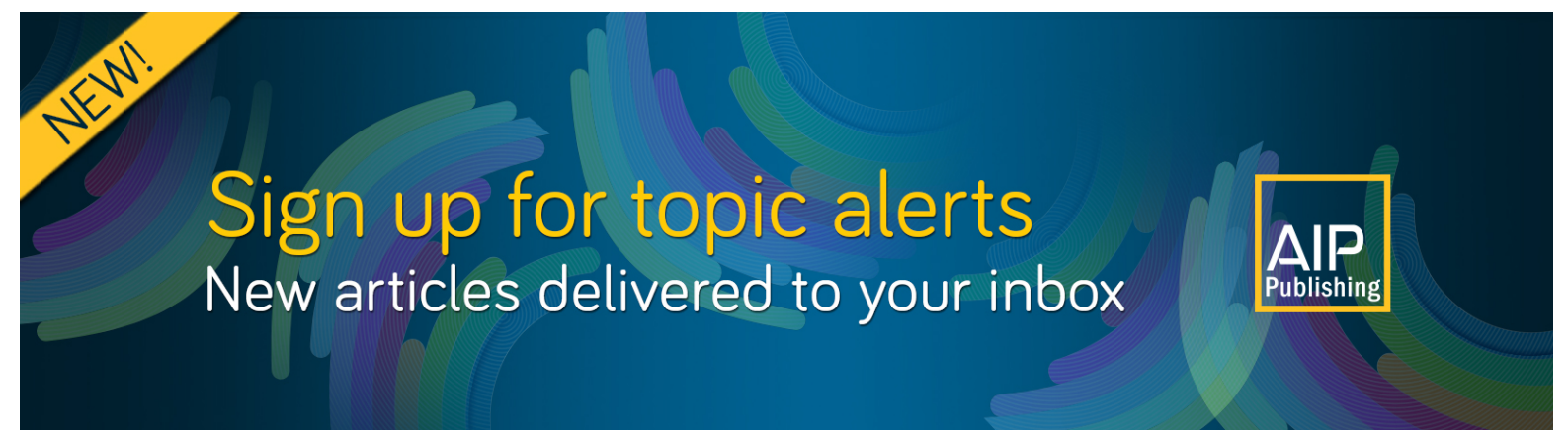




\title{
Instability of thermocapillary-buoyancy convection in droplet migration
}

\author{
Cite as: Phys. Fluids 31, 122101 (2019); doi: 10.1063/1.5125846 \\ Submitted: 30 August 2019 - Accepted: 7 November 2019 • \\ Published Online: 2 December 2019
}

Kai-Xin Hu, ${ }^{1, a), b)}$ (D) Chen-Yi Yan, ${ }^{1, b)}$ and Qi-Sheng Chen ${ }^{2,3}$

\begin{abstract}
AFFILIATIONS
${ }^{1}$ Key Laboratory of Impact and Safety Engineering, Ministry of Education, School of Mechanical Engineering and Mechanics, Ningbo University, Ningbo, Zhejiang 315211, China

${ }^{2}$ School of Engineering Science, University of Chinese Academy of Sciences, Beijing 100190, China

${ }^{3}$ Key Laboratory of Microgravity, Institute of Mechanics, Chinese Academy of Sciences, Beijing 100190, China
\end{abstract}

a) Author to whom correspondence should be addressed: hukaixin@ @ nbu.edu.cn
b) Contributions: K.-X. Hu and C.-Y. Yan contributed equally to this work.

\begin{abstract}
The instabilities of thermocapillary-buoyancy convection in droplet migration are examined by linear stability analysis. The droplet is flattened by gravity and placed on a unidirectional heated solid surface. The velocity and temperature distributions of basic flow are derived as a function of the migration velocity and the Bond number. The critical Marangoni number is obtained, which depends on the Prandtl number $(P r)$, the Bond number, and the migration velocity. The preferred modes at small and moderate $\operatorname{Pr}$ are oblique waves, which travel either upstream or downstream. For high $P r$, the preferred modes include oblique and streamwise waves, while the amplitude of temperature on the surface is much smaller than that of the hot spot in the flow region. The instability mechanism is discussed and comparisons are made with liquid layers.
\end{abstract}

Published under license by AIP Publishing. https://doi.org/10.1063/1.5125846

\section{INTRODUCTION}

A liquid droplet can be set in motion by a temperature-induced surface tension gradient when it is placed on a nonuniformly heated solid surface. This phenomenon is called the thermocapillary migration. It plays a crucial role in a variety of practical applications, such as microfluidic devices, ${ }^{1}$ material processing, ${ }^{2}$ inkjet printing, ${ }^{3}$ and so on. Therefore, abundant theoretical, numerical, and experimental studies ${ }^{2,4-7}$ have been devoted to study the nature of thermocapillary migration over the last decades.

The behavior of a two-dimensional liquid droplet on a horizontal solid surface with an imposed temperature gradient has been explored by Smith. ${ }^{8}$ The lubrication theory is used to develop an evolution equation for the shape of the droplet. Ford and Nadim ${ }^{9}$ have investigated the thermocapillary migration velocity of a thin two-dimensional droplet, having an arbitrary height profile, on a solid surface. Pratap, Moumen, and Subramanian ${ }^{5}$ have presented a lubrication theory-based model for the thermocapillary motion of spherical-cap droplets on a solid surface and experimental results on decane drops moving on a polydimethylsiloxane (PDMS) coated glass slide. Dai et al. ${ }^{10}$ have reported the development of a theoretical model and experimental investigation on the migration behavior of paraffin oil droplets induced by the unidirectional thermal gradient. They have also extended the work to the migration by an omnidirectional thermal gradient. ${ }^{1}$

In the thermocapillary migration, there is a fluid convection within the droplet. The convection driven by the thermocapillary force also appears in many other physical systems, such as crystal growth ${ }^{12}$ and dewetting. ${ }^{13}$ The instability of thermocapillary convection has been studied extensively.

Smith and Davis ${ }^{14}$ have investigated the thermocapillary instabilities of a thin-film, where a fluid layer is driven by a constant temperature gradient imposed on the interface. The oblique hydrothermal waves predicted by their model have been observed in both the experiment ${ }^{15}$ and the numerical simulation. ${ }^{16}$ Chan and Chen ${ }^{17}$ have studied the linear stability of thermocapillary fluid layers coupled with the effect of gravity for the Prandtl number $\mathrm{Pr}$ $=13.9$. Their numerical results compare favorably with the experiment by Riley and Neitzel. ${ }^{15}$ Zhang et al. ${ }^{18}$ have presented a series of three-dimensional numerical simulations on the effect of surface 
heat dissipation on thermocapillary convection of moderate Prandtl number fluid in a shallow annular pool. The sideband thermocapillary instability of a thin liquid film flowing down the outside of a hot vertical cylinder has been investigated by Davalos-Orozco. ${ }^{19}$ Kang et al. ${ }^{20}$ have presented a space experimental study on the thermocapillary convection in an open annular liquid pool. Zhong and Duan ${ }^{21}$ have experimentally investigated the thermal patterns of an evaporating sessile ethanol droplet surface under steady state conditions by varying the substrate temperature. Marangoni instabilities of a sessile droplet of $0.65 \mathrm{cSt}$ silicone oil evaporating at constant contact line mode have been experimentally investigated by Wang and Shi. ${ }^{22}$ They suggested that the Bénard-Marangoni instability does not occur in the droplet when the contact angle is too large.

Droplet dynamics under the effect of Marangoni stresses have been the subject of many other studies recently. Albernaz et al. ${ }^{23}$ have examined the Marangoni effects in a hexane droplet under evaporation and close to its critical point. The Marangoni flow and free convection during crystallization of a salt solution droplet have been experimentally investigated by Kuznetsov et al. ${ }^{24} \mathrm{Kim}$ et al. ${ }^{25}$ have considered an alcohol drop placed above a thin aqueous film. They have constructed scaling laws to predict the dewetting rates of the film by considering the Marangoni stress, viscous shear stress, and evaporation. Karapetsas et al. ${ }^{26}$ have studied the effect of contact line dynamics on the thermocapillary motion of a droplet on an inclined plate. Their results demonstrate that temperature-induced variations of the equilibrium contact angle give rise to complex dynamics. Zheng et al. ${ }^{27}$ have extended a lattice Boltzmann equation (LBE) with the continuous surface force (CSF) to simulate the thermocapillary migration of two/three dimensional deformable droplets.

The flow instability of thermocapillary migration is a fundamental problem, which is crucial for the study of flow transition in droplet migration. When the Marangoni number exceeds a critical value, the internal flow of the droplet becomes unstable. This may lead to the transition to turbulence, whose velocity and temperature are chaotic. The controllability of the migration will be reduced significantly. The stability analysis can predict the range of stable flows, which is of great practical importance for the development of the migration technique. However, so far, to the best of our knowledge, no studies have been carried out for this problem. It is highly desirable to solve it, which is the purpose of this paper. In the present work, the linear stability analysis has been performed on the thermocapillary-buoyancy convection in droplet migration on a unidirectional heated plane. The thin film model is used. ${ }^{10,11}$ The droplet is assumed to be Newtonian, and the results are presented for Prandtl numbers $0.01,1$, and 100.

This paper is organized as follows: In Sec. II, the physical model and mathematical formulations are presented. The dimensionless governing equations and the solution of the basic flow are derived. Then, in Sec. III, the critical parameters are obtained. The perturbation flow fields are displayed and the energy mechanism is studied. After that, the instability mechanism is discussed, and comparisons are made with liquid layers in Sec. IV. Finally, the conclusions are presented in Sec. V.

\section{PROBLEM FORMULATION}

We consider the case of a flat droplet placed on a horizontal and rigid plane with a constant temperature gradient in Fig. $1{ }^{10}$ Here,

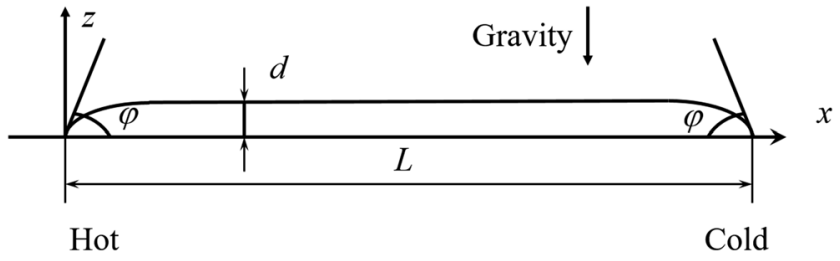

FIG. 1. Thermocapillary migration of a flat droplet on the rigid plane.

the height of the droplet $d$ is far less than the width $L$. Thus, the droplet can be seen as a thin film. $x, y$, and $z$ are the streamwise, spanwise, and wall-normal directions, respectively. The droplet is in contact with an inviscid atmosphere while $\varphi$ is the contact angle. Hence, there is also a temperature gradient on the free surface, which leads to the thermocapillary migration of the droplet.

In the presence of gravity, one would expect a droplet with an oval shape. However, the variation of $d$ is often not obvious except in the vicinity of the three-phase contact line, which can also be seen in Fig. 2 of Ref. 10. As the flow near the rim of film is not of our interest in this paper, $d$ is supposed to be a constant in the following for simplicity. With this assumption, the theoretical approach is still able to capture the main physical aspects of flow instability in thermocapillary migration.

Brochard ${ }^{28}$ has derived that when the width of the droplet is far larger than the capillary length $\hat{\kappa}^{-1}=\sqrt{\sigma_{0}^{\prime} /\left(\rho_{0} g\right)}$, the drop is flattened by gravity and forms a pancake with the thickness $d$ $=2 \hat{\kappa}^{-1} \sin (\varphi / 2)$. Here, $\sigma_{0}^{\prime}, \rho_{0}$, and $g$ are the surface tension, fluid density, and gravitational acceleration, respectively. It can be seen that $d$ has the same order as $\hat{\kappa}^{-1}$ when $\varphi$ is not too small. Thus, $L \gg \hat{\kappa}^{-1}$ as we have assumed that $L \gg d$, and the assumption of a flat surface is reasonable.

The surface tension is big enough so that the liquid surface is nondeformable. ${ }^{14}$ For simplicity, we suppose that the viscosity of the droplet is constant and the contact angle is not very sensitive to the temperature, then the difference between the left contact angle and the right one is neglected.

\section{A. Governing equations}

In this work, we assume that the surface tension is linearly related to the temperature $T: \sigma^{\prime}=\sigma_{0}^{\prime}-\gamma\left(T-T_{0}\right)$, where $\gamma$ is the negative rate of the change of surface tension with temperature. $R e$ is the Reynolds number defined as $R e=\rho U d / \mu$, where $\mu$ is the dynamic viscosity. $U$ is the characteristic velocity with the expression $U$ $=\lambda \gamma d / \mu$, where $\lambda$ is the temperature gradient of the plane. The variation of surface tension with temperature on the free surface leads to the thermocapillary effect. It can be measured by the Marangoni number $M a=\lambda \gamma d^{2} /(\chi \mu)$, which compares the thermal transport via flow due to a surface tension gradient $(\lambda \gamma)$ with thermal diffusion $(\chi)$. The migration velocity is not directly related to $M a$. We also have that $M a=R e \cdot P r$. Here, $\operatorname{Pr}$ is the Prandtl number defined as $\operatorname{Pr}=\mu /(\rho \chi) . S=\rho d \sigma_{0}^{\prime} / \mu^{2}$ is the nondimensional surface-tension number.

The magnitude of the surface deformation can be measured by the capillary number ${ }^{14}$

$$
C a=\frac{M a}{\operatorname{Pr} \cdot S}
$$


TABLE I. The scales of nondimensionalization.

\begin{tabular}{lcccc}
\hline \hline Length & Velocity & Stress & Density & Temperature \\
\hline$d=2 \sqrt{\sigma_{0}^{\prime} / \rho_{0} g} \sin (\varphi / 2)$ & $U=\lambda \gamma d / \mu$ & $F_{0}=\mu U / d$ & $\rho_{0}$ & $\lambda d$ \\
\hline \hline
\end{tabular}

For the silicone oil, $S$ and $C a$ are typically of the order of 25000 and 0.001 in the experiments, ${ }^{15}$ respectively. Therefore, $\mathrm{Ca}$ is far less than 1, indicating that the assumption of a flat free surface is reasonable. Moreover, the surface wave instability caused by thermocapillary shear stresses is not preferred when the surface-tension number $S$ is large enough. ${ }^{29}$ Hence, we only consider the thermocapillary instability in the flow with a nondeformable surface.

In the presence of gravity, the buoyancy force may have a great effect on the flow. Suppose the fluid density depends on temperature with the form $\rho=\rho_{0}\left[1-\kappa\left(T-T_{0}\right)\right]$, where $\kappa$ is the thermal expansion coefficient. The buoyancy effect can be measured by the dynamic Bond number: ${ }^{30} B o=\rho g \kappa d^{2} / \gamma$. The reason why the Bond number is chosen instead of the Rayleigh number can be explained as follows. The former compares the buoyancy to the thermocapillary force, while the latter is independent of the surface tension gradient. Therefore, we prefer the former, which is more relevant to the thermocapillary migration.

For example, $10 \mathrm{cSt}$ silicone oil ${ }^{31}$ has $\rho=0.93 \times 10^{3} \mathrm{~kg} / \mathrm{m}^{3}, v=1$ $\times 10^{-5} \mathrm{~m}^{2} / \mathrm{s}, \kappa=1 \times 10^{-3} / \mathrm{K}, \gamma=6.4 \times 10^{-5} \mathrm{~N} /(\mathrm{Km}), \chi=9.8 \times 10^{-8}$ $\mathrm{m}^{2} / \mathrm{s}, \sigma_{0}^{\prime}=19.9 \times 10^{-3} \mathrm{~N} / \mathrm{m}$, and $\operatorname{Pr}=102$. When $\varphi=140^{\circ}, \lambda$ and $g$ are on the order of $1 \mathrm{~K} / \mathrm{mm}$ and $2.5 \mathrm{~m} / \mathrm{s}^{2}$, respectively, and $d \sim O$ $(5 \mathrm{~mm}), B o \sim O(1)$, and $M a \sim O\left(2 \times 10^{3}\right)$, which are in the range we considered in this paper.

The dimensionless governing equations, which are the continuity equation, the momentum equation, and the energy equation, are given below. ${ }^{32}$ The scales of nondimensionalization are summarized in Table I. The variations of the density due to the temperature are taken into account in the momentum equations via Boussinesq's approximation $^{30}$

$$
\begin{aligned}
\nabla \cdot \mathbf{u} & =0, \\
\operatorname{Re}\left(\frac{\partial \mathbf{u}}{\partial t}+\mathbf{u} \cdot \nabla \mathbf{u}\right) & =-\nabla p+\nabla \cdot \boldsymbol{\tau}+B o \cdot T \mathbf{e}_{z}, \\
\frac{\partial T}{\partial t}+\mathbf{u} \cdot \nabla T & =\frac{1}{M a} \nabla^{2} T .
\end{aligned}
$$

Here, $\mathbf{u}=(u, v, w), p$, and $T$ stand for the velocity field, pressure, and temperature, respectively. $\tau$ is the stress. For a Newtonian fluid,

$$
\tau=\mathbf{S}
$$

where $\mathbf{S}$ is the strain-rate tensor with the form $\mathbf{S}=\nabla \mathbf{u}+(\nabla \mathbf{u})^{\mathrm{T}}$. The reference frame we choose is traveling with the droplet. ${ }^{10}$ Thus, the plane moves in the negative $x$ direction. The boundary conditions are set as follows: On the rigid plane,

$$
\left.u\right|_{z=0}=-\zeta,\left.T\right|_{z=0}=-x .
$$

Here, $\zeta$ is the nondimensional migration velocity of the droplet. In the reference frame, the flux in the $x$ direction is zero: $\int_{0}^{1} u \mathrm{~d} z=0$.
The temperature gradient of the plane is linear in $x$. On the free surface,

$$
\left.\tau_{13}\right|_{z=1}=-\left.\frac{\partial T}{\partial x}\right|_{z=1},\left.\tau_{23}\right|_{z=1}=-\left.\frac{\partial T}{\partial y}\right|_{z=1},\left.\frac{\partial T}{\partial z}\right|_{z=1}=0 .
$$

The first two equations stand for the relation between the temperature gradient and stress caused by the thermocapillary effect, while the last stands for zero heat flux.

We use the thin film model as $d \ll L$. Then, the basic flow is assumed to be parallel, while its temperature is linear in $x$ as imposed plus a distribution in $z$ as follows: ${ }^{14}$

$$
\mathbf{u}=(u(z), 0,0), T(x, z)=-x+T_{b}(z) .
$$

Then, the solutions of the basic flow can be derived as follows, and the details are presented in the Appendix:

$$
\begin{gathered}
u(z)=-\zeta\left(1-3 z+\frac{3}{2} z^{2}\right)+\left(-\frac{1}{2} z+\frac{3}{4} z^{2}\right)+\frac{B o}{4}\left(-\frac{1}{2} z+\frac{5}{4} z^{2}-\frac{2}{3} z^{3}\right), \\
T_{b}=z^{2} M a\left(\frac{\zeta}{8}(-2+z)^{2}+\frac{z}{4}\left(\frac{1}{3}-\frac{z}{4}\right)+\frac{B o}{24}\left(\frac{1}{2} z-\frac{5 z^{2}}{8}+\frac{z^{3}}{5}\right)\right) .
\end{gathered}
$$

The velocity and temperature distributions in the presence of buoyancy are displayed in Figs. 2 and 3, respectively. It can be seen that both the average of the velocity gradient and the surface temperature increase with $B o$ and $\zeta$.

Next, we investigate the range of $\zeta$. Ignoring the change of $d$ at the rim of the thin film, the dimensionless viscous resistance force $F_{v}$ can be obtained as

$$
F_{v}=\left.\int_{0}^{L / d} \tau_{x z}\right|_{z=0} \mathrm{~d} x=\frac{L}{d}\left(3 \zeta-\frac{1}{2}-\frac{1}{8} B o\right) .
$$

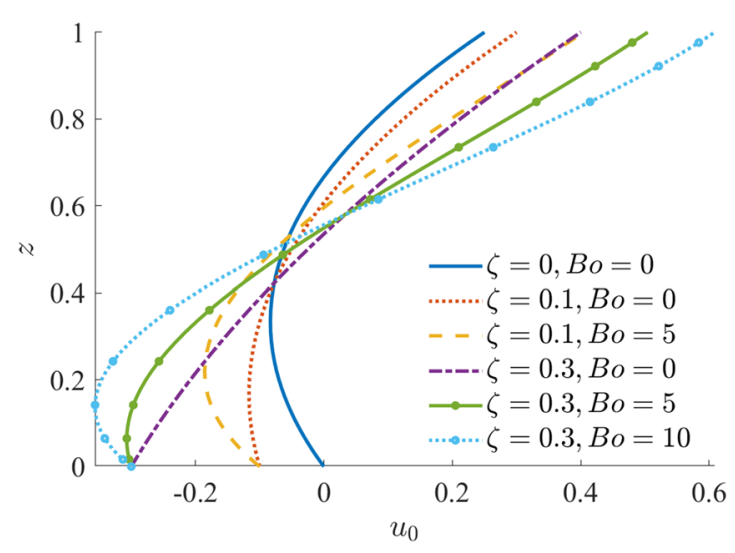

FIG. 2. The velocity distributions in the presence of buoyancy. 


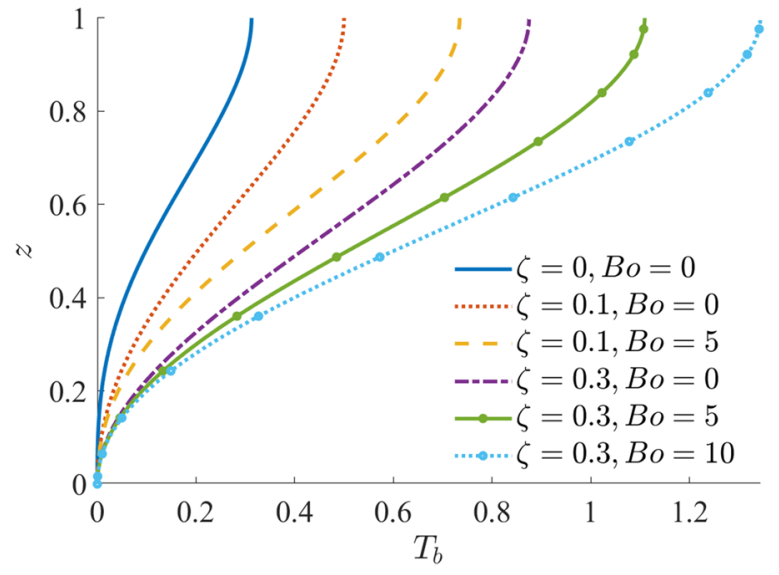

FIG. 3. The temperature distributions in the presence of buoyancy at $M a=15$.

In Fig. $1, L$ is the width of the droplet. However, we choose $d$ as the length scale, and so the nondimensional width is $L / d$. This is the reason why the integration is performed by with $0, L / d$ limits.

The driving force exerted on the droplet is $\lambda \gamma L \cos \varphi^{10}$. In order to derive its dimensionless form $F_{d}$, we should use the stress scale defined as $F_{0}=\mu U / d$. Then, it can be deduced that $F_{d}\left(F_{0} d\right)$ $=\lambda \gamma L \cos \varphi$. Substituting the expression of characteristic velocity $U=\lambda \gamma d / \mu$, we can obtain the dimensionless driving force as follows:

$$
F_{d}=\frac{L}{d} \cos \varphi
$$

where $\varphi$ is the contact angle. The contact angle of a flat water drop is $5.6^{\circ}$ at a glass substrate, ${ }^{33}$ while the contact angle of $\mathrm{Al}$ alloys to $\mathrm{SiC}$ is $157^{\circ} .^{34}$ Thus, we consider the case of the contact angle $\varphi \in\left[0^{\circ}\right.$, $\left.180^{\circ}\right]$ in this paper. There is a balance between the driving force and the viscous force $\left(F_{d}=F_{v}\right)$, and so

$$
\zeta=\frac{2 \cos \varphi+1}{6}+\frac{B o}{24} \in\left[-\frac{1}{6}+\frac{B o}{24}, \frac{1}{2}+\frac{B o}{24}\right] .
$$

\section{B. Perturbation equations}

Suppose an infinitesimal perturbation in the normal mode form is added to the basic flow

$$
\begin{gathered}
(\mathbf{u}, T, P, \boldsymbol{\tau})=\left(\mathbf{u}_{0}, T_{0}, P_{0}, \boldsymbol{\tau}_{0}\right)+\boldsymbol{\delta}, \\
\boldsymbol{\delta}=(\hat{\mathcal{u}}, \hat{v}, \hat{w}, \hat{T}, \hat{p}, \hat{\boldsymbol{\tau}}) \exp [\sigma t+\mathrm{i}(\alpha x+\beta y)] .
\end{gathered}
$$

Hereafter, the subscript 0 stands for the basic flow, and the variables without subscript 0 stand for the perturbation. $\sigma=\sigma_{r}+\mathrm{i} \sigma_{i}$, where $\sigma_{r}$ and $\sigma_{i}$ are the growth rate and frequency, respectively. The wave number, wave speed, and wave propagation angle are defined as $k=\sqrt{\alpha^{2}+\beta^{2}}, c=-\sigma_{i} / k$, and $\theta=\tan ^{-1}(\beta / \alpha)$, respectively. Due to symmetry, we shall confine ourselves to the case of $\theta \in\left[0^{\circ}, 180^{\circ}\right)$.
Substituting (2.13) and (2.14) into the governing equations (2.2)-(2.5), we can obtain the linearized perturbation equations as follows:

$$
\begin{gathered}
\mathrm{i} \alpha \hat{u}+\mathrm{i} \beta \hat{v}+D \hat{w}=0 \\
\operatorname{Re}\left(\sigma \hat{u}+\hat{w} D u_{0}+u_{0} \mathrm{i} \alpha \hat{u}\right)=-\mathrm{i} \alpha \hat{p}+\mathrm{i} \alpha \hat{\tau}_{11}+\mathrm{i} \beta \hat{\tau}_{12}+D \hat{\tau}_{13} \\
\operatorname{Re}\left(\sigma \hat{v}+u_{0} \mathrm{i} \alpha \hat{v}\right)=-\mathrm{i} \beta \hat{p}+\mathrm{i} \alpha \hat{\tau}_{12}+\mathrm{i} \beta \hat{\tau}_{22}+D \hat{\tau}_{23} \\
\operatorname{Re}\left(\sigma \hat{w}+u_{0} \mathrm{i} \alpha \hat{w}\right)=-D \hat{p}+\mathrm{i} \alpha \hat{\tau}_{13}+\mathrm{i} \beta \hat{\tau}_{23}+D \hat{\tau}_{33}+B o \hat{T} \\
M a\left(\hat{u} \frac{\partial T_{0}}{\partial x}+\hat{w} \frac{\partial T_{0}}{\partial z}+u_{0} \mathrm{i} \alpha \hat{T}\right)+\left(\alpha^{2}+\beta^{2}\right) \hat{T}-D^{2} \hat{T}=-\sigma M a \hat{T} \\
\hat{\tau}_{11}-(2 \mathrm{i} \alpha \hat{u})=0, \hat{\tau}_{12}-(\mathrm{i} \alpha \hat{v}+\mathrm{i} \beta \hat{u})=0, \hat{\tau}_{13}-(D \hat{u}+\mathrm{i} \alpha \hat{w})=0 \\
\hat{\tau}_{22}-(2 \mathrm{i} \beta \hat{v})=0, \hat{\tau}_{23}-(\mathrm{i} \beta \hat{w}+D \hat{v})=0, \hat{\tau}_{33}-(2 D \hat{w})=0
\end{gathered}
$$

Here, $D$ stands for $\mathrm{d} / \mathrm{d} z$. The stress tensor is solved in (2.20) and (2.21) separately from the velocity. The advantage is that this form of equation can be easily generalized to non-Newtonian fluids, where we only need to change the constitutive equations [(2.20) and (2.21)], while other equations [(2.15)-(2.19)] remain the same.

In (2.16)-(2.18), $\bar{p}$ can be eliminated, and the following equations are derived:

$$
\begin{aligned}
& \operatorname{Re}\left[\beta\left(\hat{w} D u_{0}+u_{0} \mathrm{i} \alpha \hat{u}\right)-\alpha\left(u_{0} \mathrm{i} \alpha \hat{v}\right)\right]-\beta\left(i \alpha \bar{\tau}_{11}+\mathrm{i} \beta \hat{\tau}_{12}+D \hat{\tau}_{13}\right) \\
& +\alpha\left(\mathrm{i} \alpha \hat{\tau}_{12}+\mathrm{i} \beta \hat{\tau}_{22}+D \hat{\tau}_{23}\right)=-\sigma R e(\beta \hat{u}-\alpha \hat{v}), \\
& \operatorname{Re} \alpha\left(D \hat{w} \cdot D u_{0}+\hat{w} D^{2} u_{0}+D u_{0} \cdot \mathrm{i} \alpha \hat{u}+u_{0} \mathrm{i} \alpha D \hat{u}\right) \\
& +\operatorname{Re} \beta\left(D u_{0} \cdot \mathrm{i} \alpha \hat{v}+u_{0} \mathrm{i} \alpha D \hat{v}\right)-\operatorname{Re}^{2} k^{2}\left(u_{0} \mathrm{i} \alpha \hat{w}\right) \\
& -\left(\mathrm{i} \alpha^{2} D \bar{\tau}_{11}+2 \mathrm{i} \alpha \beta D \bar{\tau}_{12}+\alpha D^{2} \hat{\tau}_{13}+\mathrm{i} \beta^{2} D \bar{\tau}_{22}+\beta D^{2} \hat{\tau}_{23}\right) \\
& +\mathrm{i} k^{2}\left(\mathrm{i} \alpha \bar{\tau}_{13}+\mathrm{i} \beta \hat{\tau}_{23}+D \hat{\tau}_{33}+B o \cdot \hat{T}\right) \\
& =-\sigma \operatorname{Re}\left(\alpha D \hat{u}+\beta D \hat{v}-\mathrm{i} k^{2} \hat{w}\right) \text {. }
\end{aligned}
$$

The boundary conditions for the perturbation are determined as follows:

$$
\begin{gathered}
\hat{u}=\hat{v}=\hat{w}=\hat{T}=0, z=0, \\
\hat{\tau}_{13}+\mathrm{i} \alpha \hat{T}=0, \hat{\tau}_{23}+\mathrm{i} \beta \hat{T}=0, \hat{w}=0, D \hat{T}=0, z=1 .
\end{gathered}
$$

In order to obtain the eigenvalue $\sigma$, the Chebyshev collocation method $^{35}$ is used. $N_{c}$ Gauss-Lobatto points are set in the flow region $z=\left(1-\cos \left(\frac{j \pi}{N_{c}+1}\right)\right) / 2, \quad j=1 \sim N_{c}$ for Eqs. (2.15)-(2.21), while 2 points are set at the boundaries $z=0,1$ for Eqs. (2.24) and (2.25). 
TABLE II. The eigenvalues of neural modes computed by different numbers of nodes.

\begin{tabular}{lccccc}
\hline \hline & \multicolumn{2}{c}{ Case 1} & & \multicolumn{2}{c}{ Case 2 } \\
\cline { 2 - 3 } \cline { 5 - 6 }$N_{c}$ & $\sigma_{r}\left(\times 10^{-6}\right)$ & $\sigma_{i}\left(\times 10^{-1}\right)$ & & $\sigma_{r}\left(\times 10^{-6}\right)$ & $\sigma_{i}\left(\times 10^{-2}\right)$ \\
\hline 70 & 1.819430 & 4.299003 & & 3.736183 & 6.834245 \\
80 & 1.819234 & 4.299003 & & 3.736175 & 6.834245 \\
90 & 1.819271 & 4.299003 & & 3.736152 & 6.834245 \\
\hline \hline
\end{tabular}

The perturbation quantities are expanded in Chebyshev polynomials as

$$
\hat{u}=\sum_{j=1}^{N_{c}+1} a_{j} A_{j-1}(\bar{z}) .
$$

Here, $\bar{z}=1-2 z, A_{j-1}(\hat{z})=\cos \left[(j-1) \cos ^{-1}(\hat{z})\right]$ is the $(j-1)$ th Chebyshev polynomial, and $a_{j}$ is the coefficient. The general eigenvalue problem can be solved in the form of $\mathbf{W v}=\sigma \mathbf{Z v}$, where $\mathbf{W}$, $\mathbf{Z}$ are two matrices, and $\mathbf{v}$ is the eigenvector. The eigenvalues are obtained by using the QZ algorithm available in the Matlab-software package. In the computation, the results are sufficiently accurate when $N_{c}=70-90$. The convergence can be seen in Table II.

Here, case 1 has $\operatorname{Pr}=1, \zeta=0.3, B o=5, M a_{c}=124.13, k=1.896$, and $\theta=89^{\circ}$, while case 2 has $\operatorname{Pr}=0.01, \zeta=0.6, B o=10, M a_{c}=15.43$, $k=1.098$, and $\theta=88^{\circ}$.

\section{NUMERICAL RESULTS}

We compute the neutral Marangoni number $M a_{N}$ of neutral mode $\left(\sigma_{r}=0\right)$. The critical Marangoni number $M a_{c}$ is defined as the global minimum of neutral Marangoni numbers for all $(\alpha, \beta)$,

$$
M a_{c}=\min _{\alpha, \beta} M a_{N}(\zeta, P r)
$$

When the Marangoni number is below the critical value, $M a_{c}$, the flow is linear stable for any normal mode perturbation. In contrast, there can be an unstable mode in the flow when $M a>M a_{c}$.

In the computation, we find there are two different kinds of preferred modes (see Fig. 4), which are the streamwise wave $\left(\theta=0^{\circ}\right)$ and the oblique wave $\left(\theta \neq 0^{\circ}, 90^{\circ}\right)$, respectively. The counter-rotating rolls are arranged periodically for both of them.

The results at $\operatorname{Pr}=0.01, \operatorname{Pr}=1$, and $P r=100$ are presented in Secs. III A-III C, respectively. The perturbation fields of preferred

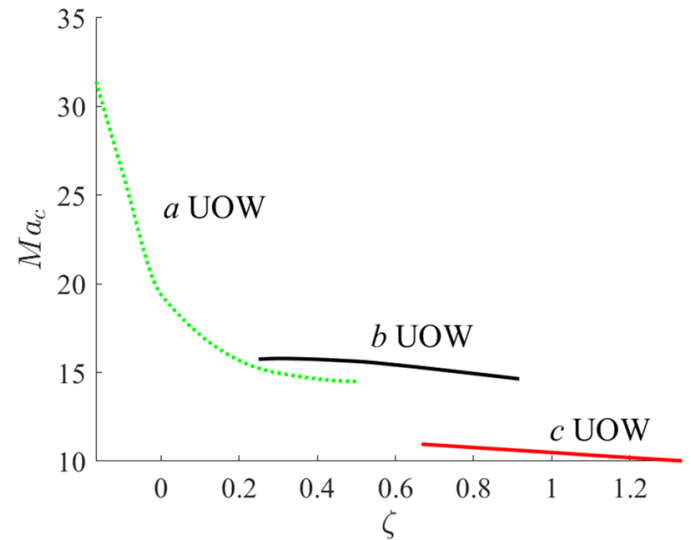

FIG. 5. The variation of $M a_{c}$ with $\zeta$ and $B o$ at $\operatorname{Pr}=0.01$. Here, UOW stands for upstream oblique waves $\left[\theta \in\left(90^{\circ}, 180^{\circ}\right)\right]$. The curves correspond to (1) $B 0=0$, oblique wave (a); (2) $B 0=10$, oblique wave (b); and (3) $B 0=20$, oblique wave (c).

modes are displayed in Sec. III D, and the energy analysis is performed in Sec. III E.

\section{A. $\operatorname{Pr}=0.01$}

The variation of $M a_{c}$ with $\zeta$ and $B o$ at $P r=0.01$ is displayed in Fig. 5. When $B o=0$ [curve (a)], there is a significant decrease in $M a_{c}$ with $\zeta$. When $B o=10$ and 20, [curves (b) and (c)], $M a_{c}$ decreases slightly with the increase in $\zeta$. However, the variation of $M a_{c}$ with $B o$ is nonmonotonic. $M a_{c}$ increases with $B o$ at $\zeta=0.4$ but decreases at $\zeta=0.8$. The preferred modes are always the upstream oblique wave $\left[\theta \in\left(90^{\circ}, 180^{\circ}\right)\right]$.

The wave number, the propagation angle, and the wave speed corresponding to the modes in Fig. 5 are displayed in Fig. 6. It can be seen that the wave numbers are on the order of $1 . k$ is nearly a constant at $B o=10$ and 20 . The oblique waves are upstream and close to spanwise traveling waves. The propagation angle tends to $90^{\circ}$ when $B o$ and $\zeta$ increase. The wave speed significantly increases with $B o$.

\section{B. $\operatorname{Pr}=1$}

The neutral curves at $\operatorname{Pr}=1$ are displayed in Fig. 7. It can be found that $M a_{c}$ always decreases with the increase in $\zeta$ but increases with $B o$. The preferred modes include upstream and downstream oblique waves.

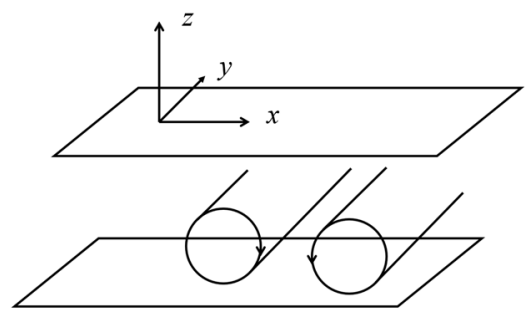

(a)

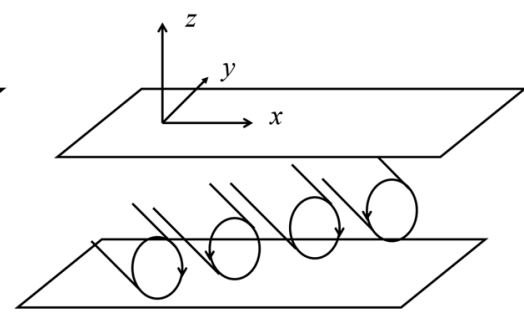

(b)
FIG. 4. The schematic of preferred modes: (a) streamwise wave and (b) oblique wave. 


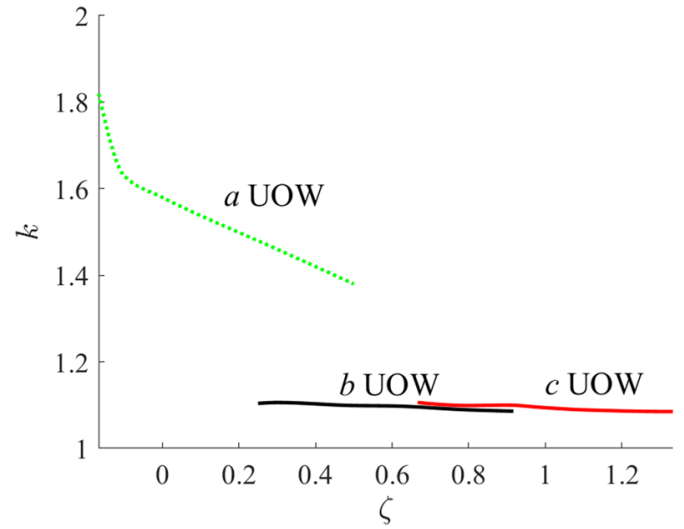

(I)

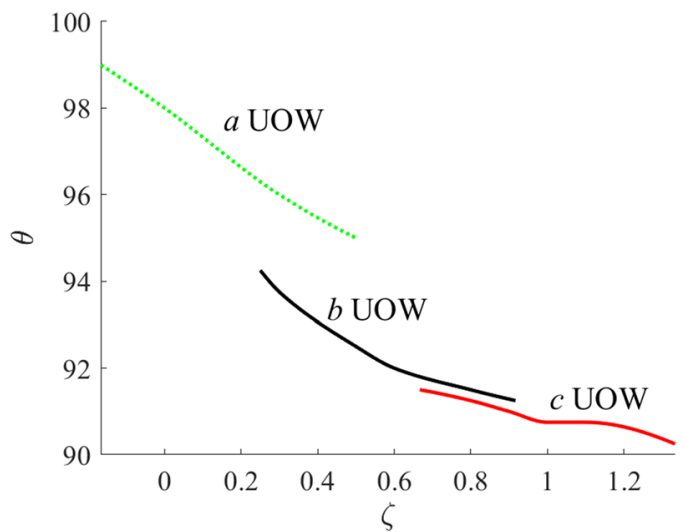

(II)

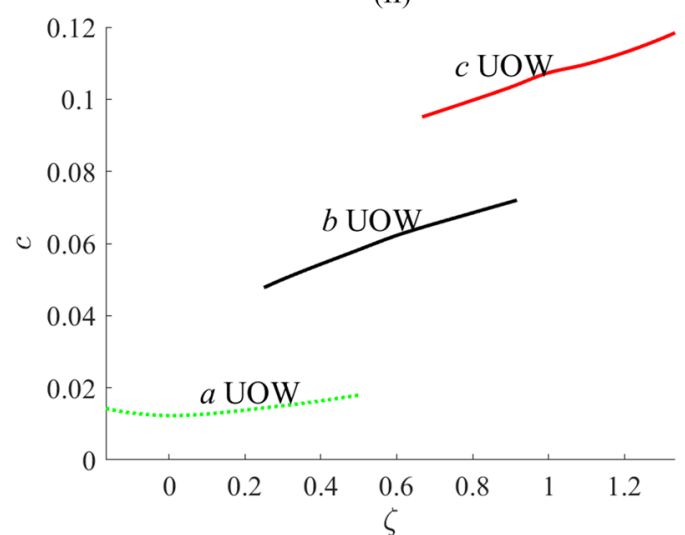

(III)

FIG. 6. The (I) wave number, (II) wave propagation angle, and (III) wave speed corresponding to the modes in Fig. 5.

The wave number, the wave propagation angle and the wave speed corresponding to the modes in Fig. 7 are displayed in Fig. 8. It can be found that the wave numbers are the order of 2 . The preferred modes change from upstream to downstream when $B o$ is large enough. The variation of the wave speed with $\zeta$ is almost

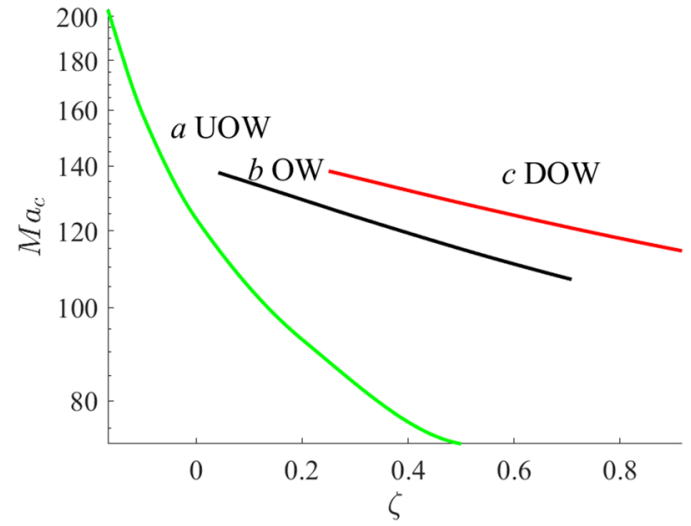

FIG. 7. The variation of $M a_{c}$ with $\zeta$ and $B o$ at $P r=1$. Here, DOW stands for downstream oblique waves $\left[\theta \in\left(0^{\circ}, 90^{\circ}\right)\right]$. Curve $(b)$ changes from upstream to downstream when $\zeta$ increases. So we use $O W$ to stand for the oblique wave (b). The curves correspond to (1) $B o=0$, oblique wave (a); (2) $B o=5$, oblique wave (b); and (3) Bo = 10, oblique wave (c).

linear. The increase in the wave speed with $B o$ is obvious, which is similar to the case at $\operatorname{Pr}=0.01$.

\section{C. $\operatorname{Pr}=100$}

The neutral curves at $\mathrm{Pr}=100$ are displayed in Fig. 9. It can be seen that $M a_{c}$ significantly increases with $B o$. When $B o=0, M a_{c}$ obviously decreases with $\zeta$, which is similar to the cases at $\operatorname{Pr}=0.01$ and $P r=1$, and the preferred modes include oblique and streamwise waves. When $B o=5$ and 10 , all preferred modes are streamwise waves, and the variation of $M a_{c}$ with $\zeta$ is little.

The wave number, the wave propagation angle, and the wave speed corresponding to the modes in Fig. 9 are displayed in Fig. 10. Both the wave number and wave speed increase with Bo significantly. The preferred modes at $B o=0$ is upstream, while those at $B o \geq 5$ are downstream. The wave speed increases linearly with $\zeta$ when $B o \geq 5$.

\section{The perturbation flow field}

The streamlines and isothermals of the preferred mode at $P r=0.01$ are displayed in Fig. 11. $T_{s}$ is the temperature perturbation on the surface. The hot spots are on the surface in Figs. 11(I) and 11(II). These differ from the cases in the liquid layer, ${ }^{36}$ where the isothermals are nearly vertical at small Prandtl numbers. The difference is due to the boundary condition. There is zero heat flux on the rigid plane for the liquid layer, while the temperature perturbation is zero at the position $z=0$ in this paper [see (2.24)].

The perturbation flow field at $P r=1$ is displayed in Fig. 12 . When $B o=0$, there are hot spots in the middle region $(z=0.5)$. The fluid at the position $(z=0.5$, phase $=4)$ is heated, as the vertical down flow brings hotter fluid from the surface. So the temperature in this region increases and the wave travels upstream. When $B o=10$, the fluid at the position $(z=0.5$, phase $=2.5)$ is heated by the vertical down flow, and the wave travels downstream. The streamlines near the hot spot are clockwise and close to the isothermals. 


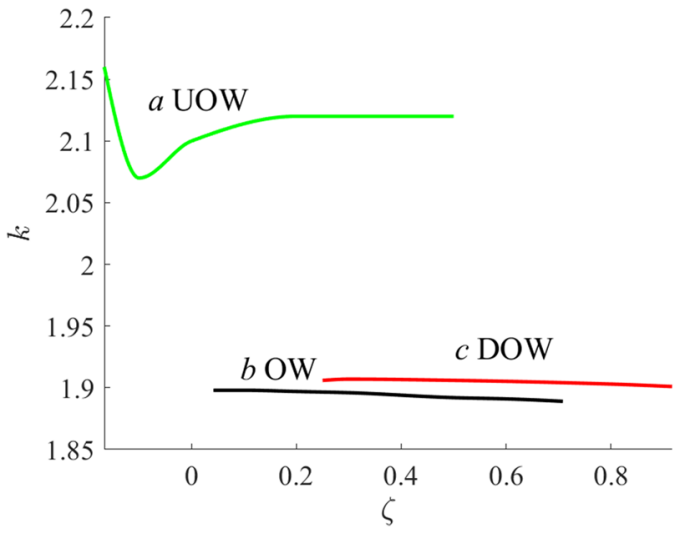

(I)

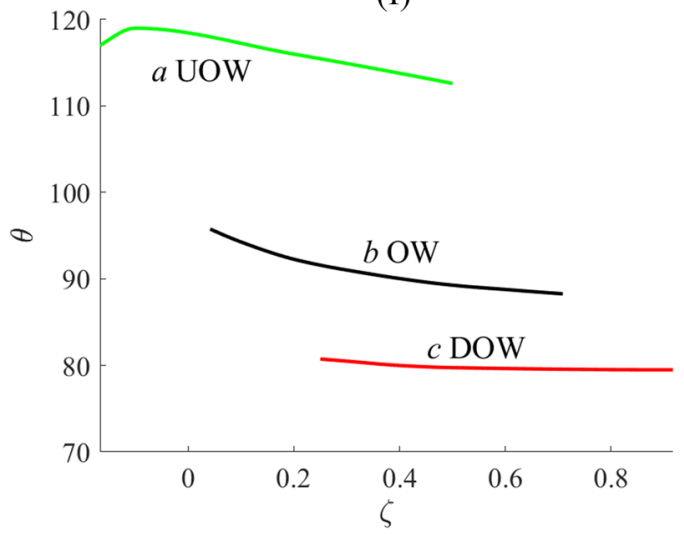

(II)

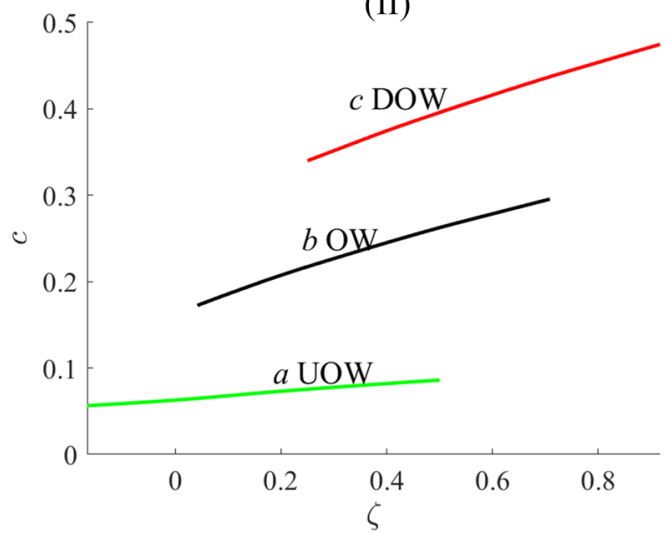

(III)

FIG. 8. The (I) wave number, (II) wave propagation angle, and (III) wave speed corresponding to the modes in Fig. 7.

The streamlines and isothermals of the preferred mode at $\mathrm{Pr}$ $=100$ are displayed in Fig. 13. The downstream streamwise wave in Fig. 13 has hot spots in the interior. The temperature of the fluid at the position $(z=0.5$, phase $=3.2)$ will be decreased by the vertical upflow, as it brings cooler fluid from the bottom. So the wave travels upstream.

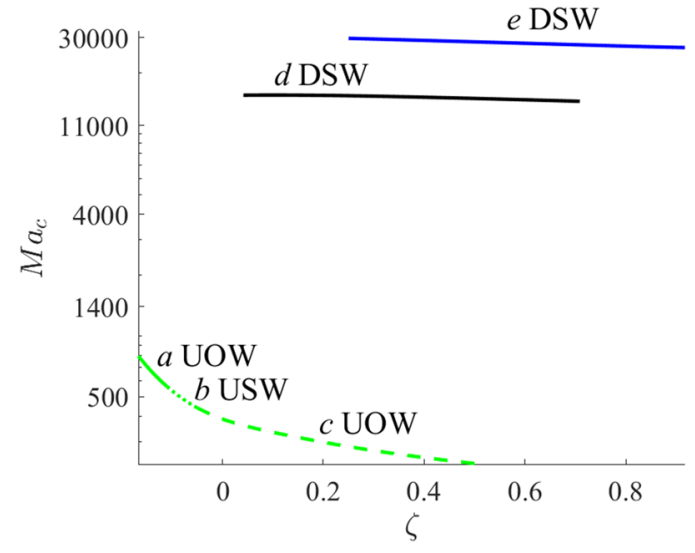

FIG. 9. The variation of $M a_{c}$ with $\zeta$ and $B o$ at $P r=100$. Here, USW and DSW stand for upstream $\left(\theta=180^{\circ}\right)$ and downstream $\left(\theta=0^{\circ}\right)$ streamwise waves, respectively. The curves correspond to (1) $B O=0$, oblique wave $[(a)$ and (c)]; (2) $B O=0$, streamwise wave (b); (3) $B o=5$, streamwise wave (d); and (4) $B 0=10$, streamwise wave (e).

\section{E. Energy analysis}

The energy mechanism is studied in this section. The rate of change for perturbation energy can be written as follows: ${ }^{37,38}$

$$
\begin{aligned}
\frac{\partial E_{k i n}}{\partial t}= & -\frac{1}{2 R e} \int_{V}(\boldsymbol{\tau}: \mathbf{S}) \mathrm{d} \forall+\frac{1}{R e} \int_{S} \mathbf{u} \cdot \boldsymbol{\tau} \cdot \mathbf{n} \mathrm{ds} \\
& -\int_{V} \mathbf{u} \cdot\left((\mathbf{u} \cdot \nabla) \mathbf{u}_{0}\right) \mathrm{d} \forall+\int_{V}\left(\frac{B o}{R e} T \mathbf{e}_{3} \cdot \mathbf{u}\right) \mathrm{d} \forall \\
= & -N+M+I+G,
\end{aligned}
$$

where $N$ is the viscous dissipation, $M$ is the work done by Marangoni forces on the surface, $I$ is the interaction between the perturbation flow and the basic flow, and $G$ is the work done by gravity. ${ }^{30}$ Here, the subscripts $V$ and $S$ stand for the volume and surface integral, respectively.

The terms of perturbation energy growth at different Prandtl numbers and Bond numbers are displayed for neutral modes in Fig. 14. As $\sigma_{r}=0$, the sum of three terms in the table has $(M+I+G) / N=1$. It can be found that either $I$ or $M$ is the main energy source, while $G$ is not important. $M$ is dominant at $\operatorname{Pr}=1$ and 100, while the importance of $M$ depends on $B o$ and $\zeta$ at $\operatorname{Pr}=0.01$.

\section{DISCUSSION}

\section{A. Comparison of the droplet with liquid layers}

First, we want to compare the droplet with liquid layers in the following. The basic flow in the liquid layer ${ }^{30}$ is the same as the case of $\zeta=0$ in (2.9), and so the migrating droplet seems similar to a thin film with a moving bottom wall. However, there are three main differences between them. First, their basic flow and boundary conditions are different. The migration velocity of the droplet depends on the contact angle, and so the velocity distribution is a function of both $B o$ and $\zeta$ in (2.9). In contrast, the velocity distribution in the thin film layer only depends on $B o .{ }^{30}$ Meanwhile, the droplet is 


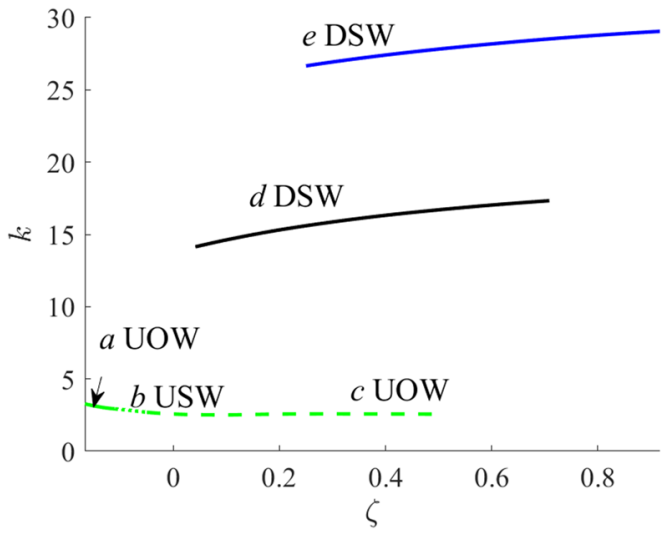

(I)

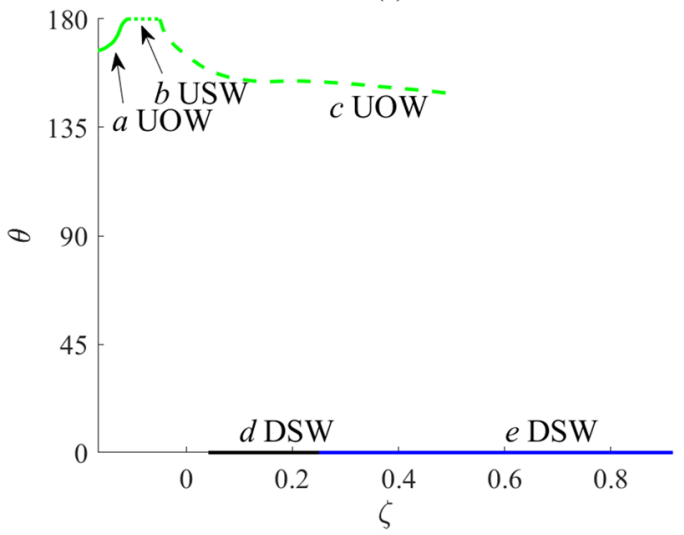

(II)

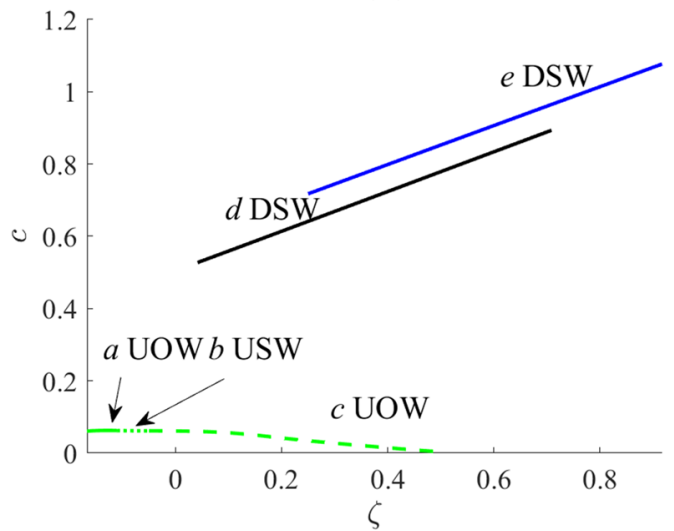

(III)

FIG. 10. The (I) wave number, (II) wave propagation angle, and (III) wave speed corresponding to the modes in Fig. 9.

placed on a nonuniformly heated solid surface, and the temperature perturbation on the wall is zero in $(2.24) .{ }^{8}$ However, the wall of the film layer has zero heat flux, ${ }^{14,16,17}$ and sometimes, the temperature perturbation on the wall is not zero. 30,35

Second, in liquid layers of Newtonian fluids at $\operatorname{Pr}=13.9^{17}$ and viscoelastic fluids at $\operatorname{Pr}=100,0.02,{ }^{30}$ the gravity only leads to
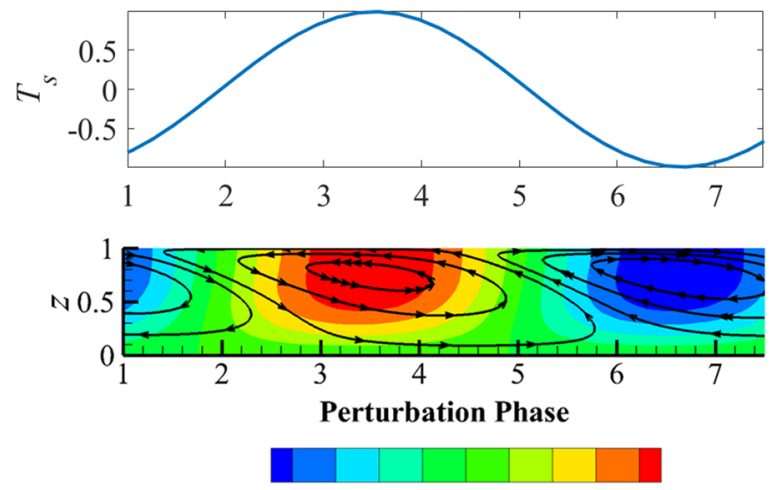

$T: \quad-0.8-0.6-0.4-0.2 \quad 0 \quad 0.20 .4 \quad 0.6 \quad 0.8$

(I)
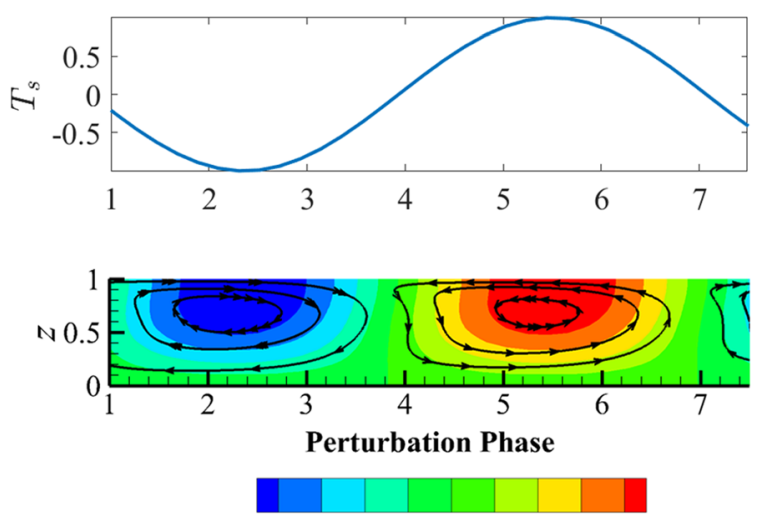

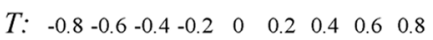

(II)

FIG. 11. The perturbation flow field of the preferred mode at $\operatorname{Pr}=0.01$ : (I) upstream oblique wave $(B O=0, \zeta=0.3)$ and (II) upstream oblique wave $(B O=10, \zeta=0.8)$.

the buoyancy. The cases studied in these works can be degenerated to that at $g=0$. However, besides buoyancy, the gravity has another important effect in this paper that makes the drop flat and form a pancake with thickness $d=2 \sqrt{\sigma_{0}^{\prime} /\left(\rho_{0} g\right)} \sin (\varphi / 2)$. Thus, the assumption of a flat surface will be invalid if $g \rightarrow 0$. In addition, $d$ is not an independent parameter, which differs from the case in liquid layers.

Third, the buoyancy effect on the stability for the liquid layer is also different from that of the droplet. In Refs. 17 and 30, the buoyancy often makes the flow more stable at high Pr. This conclusion also holds for the droplet. However, for small $P r$, the buoyancy is destabilizing in the liquid layer, ${ }^{30}$ while the variation of $M a_{c}$ with $B o$ is nonmonotonic for the droplet (see Fig. 5).

\section{B. Instability mechanism and the properties at different Prandtl numbers}

We will then discuss the instability mechanism and the properties of preferred modes. The cases of three Prandtl numbers are discussed and compared. 

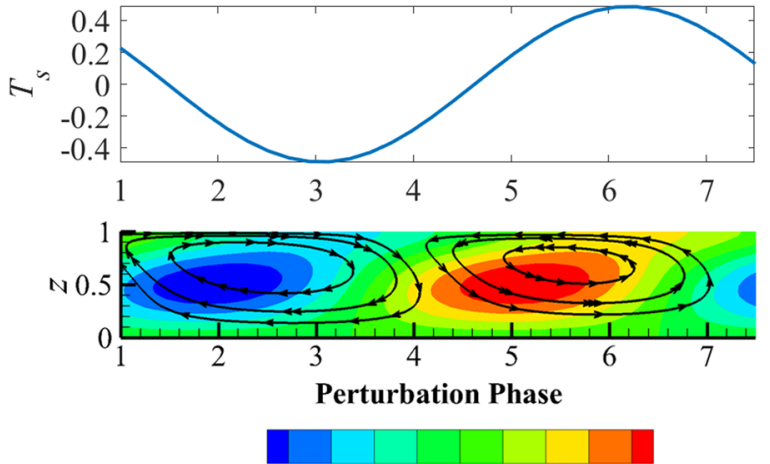

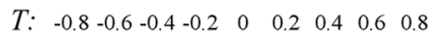

(I)
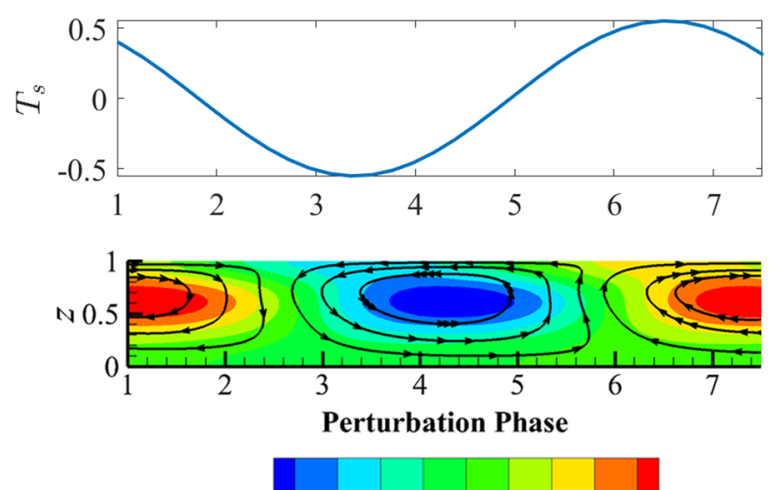

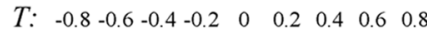

(II)

FIG. 12. The perturbation flow field at $\operatorname{Pr}=1$ : (I) upstream oblique wave $(B 0=0$, $\zeta=0.3)$ and (II) downstream oblique wave $(B o=10, \zeta=0.7)$.

\section{1. $\operatorname{Pr}=0.01$}

The modes in this work have similar mechanisms as the hydrothermal wave. ${ }^{36}$ For small $\mathrm{Pr}, \mathrm{Smith}^{36}$ has suggested that the
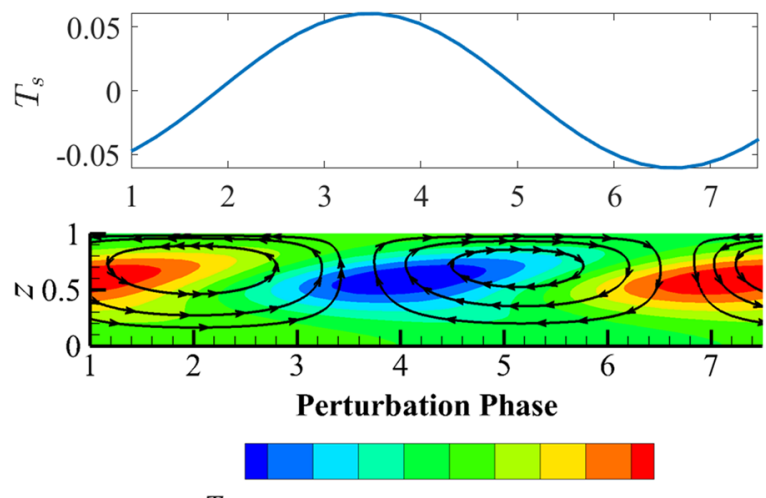

$T: \quad-0.8-0.6-0.4-0.2 \quad 0 \quad 0.20 .4 \quad 0.6 \quad 0.8$

FIG. 13. The perturbation flow field at $\operatorname{Pr}=100$ : streamwise wave $(B 0=0$, $\zeta=-0.1)$.

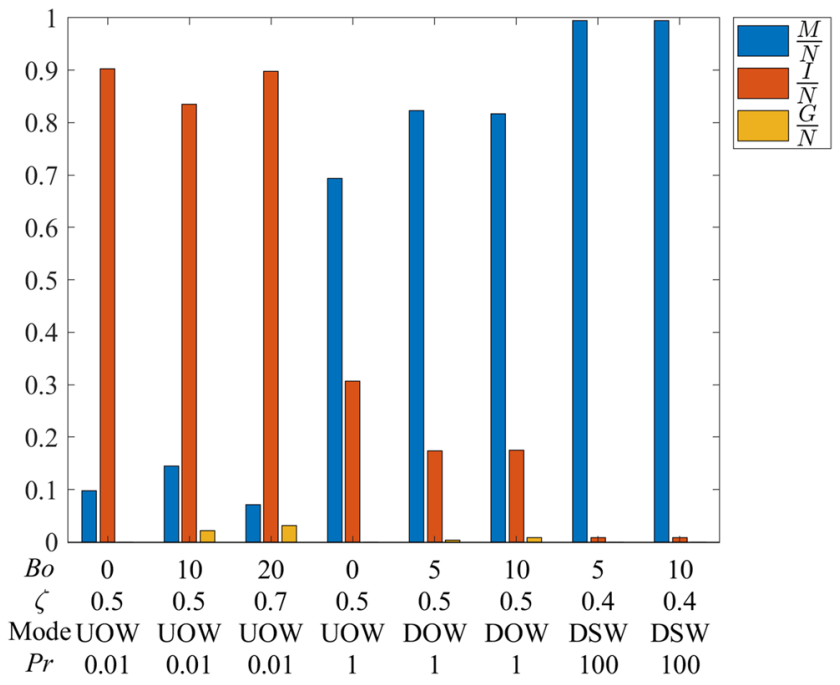

FIG. 14. The terms of perturbation energy growth at different Prandtl numbers and Bond numbers for neutral modes.

key to the mechanism is the inertial-driven streamwise flow for the return flow in the liquid layer. The cold spot on the surface is mainly caused by the horizontal convective cooling $\left(\hat{u} \frac{\partial T_{0}}{\partial x}\right)$. This is also true for the droplet at $B o=0$. However, the vertical convection $\left(\hat{w} \frac{\partial T_{0}}{\partial z}\right)$ can also cool the cold spot and will be important for the temperature field when the vertical gradient of temperature for the basic flow $\frac{\partial T_{0}}{\partial z}$ is large enough. As $\frac{\partial T_{0}}{\partial z}$ significantly increases with $B o$ and $\zeta$ (see Fig. 3), $M a_{c}$ decreases with $\zeta$ and the flow becomes more unstable when $B o$ is large enough (see Fig. 5).

\section{2. $\operatorname{Pr}=1$}

For $\operatorname{Pr}=1, M a_{c}$ always decreases with the increase in $\zeta$ but increases with $B o$ (see Fig. 7). The reason can be explained as follows. The computation shows that the vertical convection $\left(\hat{w} \frac{\partial T_{0}}{\partial z}\right)$ is dominant for the temperature field at $\operatorname{Pr}=1$. Figure 3 shows that $\frac{\partial T_{0}}{\partial z}$ increases with $\zeta$. Therefore, the flow becomes more unstable when $\zeta$ increases. Suppose a neutral mode of $B o=0$ in Fig. 12(I) is added in the basic flow at $B o>0$, the down flow near the hot spot leads to $G<0$ and stabilizes the flow, and so a higher $M a_{c}$ is needed when $B o$ increases.

\section{3. $\operatorname{Pr}=100$}

For $\operatorname{Pr}=100$, the instability is closely related to the heat convection by the basic flow $\left(u_{0} \mathrm{i} \alpha \hat{T}\right)$. Its increase with the wave number can make the perturbation more unstable. This is the reason why the wave numbers corresponding to the preferred mode in Fig. 10(I) are much higher than those at $\operatorname{Pr}=1$ and $\operatorname{Pr}=0.01$ [see Figs. 6(I) and 8(I)]. It also agrees with the results in Ref. 30, where the modes with high wave numbers are excited when $B o$ is large enough. The reason why $M a_{c}$ increases with $B o$ is similar to the that at $\operatorname{Pr}=1$.

We want to compare $k, \theta$, and $c$ at different $\operatorname{Pr}$. The wave numbers are $O(1), O(2)$, and $O(10)$ at $\operatorname{Pr}=0.01,1$, and 100, respectively [see Figs. 6(I), 8(I), and 10(I)]. However, there are only quantitative 
variations of $k$ with $\zeta$. The wave propagation angle depends highly on $\operatorname{Pr}$. For $\operatorname{Pr}=0.01$, the preferred modes are upstream oblique waves [see Fig. 6(II)]. For $\operatorname{Pr}=1$, the preferred modes change from upstream oblique waves to downstream oblique waves when $B o$ is large enough [see Fig. 8(II)]. In contrast, for $\operatorname{Pr}=100$, the preferred mode includes oblique and streamwise waves [see Fig. 10(II)]. The preferred modes at $B o=0$ are upstream, while those at $B o \geq 5$ are downstream. The wave speed always increases with $B o$, while its variation with $\zeta$ is nonmonotonic [see Figs. 6(III), 8(III), and 10 (III)].

We can compare the perturbation fields at different $P r$. In Fig. $13(\operatorname{Pr}=100)$, the amplitude of $T_{s}$ is much smaller than that of the hot spot; in Fig. $12(\operatorname{Pr}=1)$, the amplitude of $T_{s}$ is about half of that of the hot spot; while in Fig. $11(\operatorname{Pr}=0.01)$, their amplitudes are very close. The reason can be explained as follows: In most cases, the hot spot is in the interior of the droplet. As the heat conduction is important at small $\mathrm{Pr}$, the surface can be heated by conduction quickly, and so the amplitude of $T_{s}$ is close to that of the hot spot. In contrast, the surface is mainly heated by convection at high $P r$, and the conduction from the hot spot is limited. The vertical convection is very small as $|\hat{w}|$ tends to zero near the surface; thus, the amplitude of $T_{s}$ is far less than that of the hot spot. For moderate $P r$, the importance of convection is comparable with that of conduction, and so the amplitude of $T_{s}$ is less than that of the hot spot but larger than that at high $P r$.

The energy analysis shows that, for $\operatorname{Pr}=0.01, I$ is the most important energy source for the perturbation. In contrast, $M$ is often the main energy source for the liquid layer. ${ }^{32}$ When $B o$ is large enough, $G$ is not negligible anymore. For $P r=1, M$ becomes the main energy source, $I$ is still important, while $G$ is very small. For $\operatorname{Pr}=100$, both $I$ and $G$ are negligible, and so the thermocapillary force is the driving force for the perturbation, while the perturbation stresses cause damping.

\section{Comparisons with experiments}

We want to make a comparison of this work with experimental studies. There are many experiments of internal flows in heated drops. The theoretically predicted Marangoni flows are observed in Refs. 39 and 40. For thermocapillary migration, many authors have made comparisons between the experiments and theoretical descriptions. ${ }^{5,10,41}$ As their models are also used here, we can compare our results with their expressions. When $B o=0$, the velocity distribution (2.9a) coincides with (8) of Pratap et al., ${ }^{5}$ and the migration velocity (2.12) is the same as (20) of Dai et al. ${ }^{10}$ In Ref. 5, the theoretical prediction of radius is comparable with the experiment value, while in Ref. 10, the experimental migration velocity fits the numerical results well. These ensure the validity of the model and velocity distribution we used in this paper.

Sefiane et al. ${ }^{42}$ have observed hydrothermal waves in sessile drops, where the thermocapillary convection is caused by spatially nonuniform evaporative flux. The temperature gradient on the surface is self-generated in this experiment, while that of thermocapillary migration in this paper is imposed. However, their instability mechanisms are similar.

Then, we want to discuss the contact angle, which is an important factor in the droplet migration. In theoretical analysis, both the migration speed and thickness depend on $\varphi$ [see (2.12) and
Brochard's formula: $\left.{ }^{28} d=2 \hat{\kappa}^{-1} \sin (\varphi / 2)\right]$. In experiments, the influences of the contact angle are complex. For example, the contact angle hysteresis appears in the thermocapillary motion, ${ }^{5,41,43}$ where the advancing and receding contact angles $\left(\varphi_{a}, \varphi_{r}\right)$ are different. However, typical contact angle hysteresis is $\tilde{\delta}=\cos \varphi_{a}-\cos \varphi_{r}$ $=1.5 \times 10^{-2}$ for silicone oils and $\tilde{\delta}=10^{-2}$ for $\mathrm{n}$-alkanes, while the variations of $\varphi_{a}, \varphi_{r}$ with temperature are extremely small. ${ }^{41}$ Hence, we believe that contact angle hysteresis will not affect the flow instability qualitatively, and our simplifying assumption $\left(\varphi_{a}=\varphi_{r}=\varphi\right)$ is still valid. Pratap et al. ${ }^{5}$ have observed that the spherical drops in thermocapillary motion are elongated in the direction on the solid surface that is perpendicular to that of motion. It was attributed to the variation of the contact angle around the periphery of the drop. This phenomenon will make the internal flow of the spherical drop more similar to the flow in a two-dimensional droplet, which is the model we used in this paper.

\section{CONCLUSION}

In this paper, the linear stability analysis is performed on the thermocapillary-buoyancy convection in droplet migration. The conclusions are drawn as follows:

For the basic flow, the temperature difference between the surface and the bottom always increases with the migration velocity $\zeta$ and the Bond number Bo. The critical Marangoni number $M a_{c}$ always decreases with $\zeta$. For small and moderate $P r$, the preferred modes are oblique waves, which travel either upstream or downstream. For high $\mathrm{Pr}$, the preferred mode includes oblique and streamwise waves, while the amplitude of temperature on the surface is much smaller than that of the hot spot in the flow region.

The buoyancy effect on the instability depends on the Prandtl number. For small $P r$, the variation of $M a_{c}$ with $B o$ is nonmonotonic, and the perturbation energy mainly comes from the basic flow. For high $\mathrm{Pr}, \mathrm{Ma}_{\mathrm{c}}$ increases with $\mathrm{Bo}$ significantly, and the work done by Marangoni forces becomes the main energy source. The preferred modes with high wave numbers are excited when $B o$ is large enough. For moderate $\mathrm{Pr}, M a_{c}$ increases with $\mathrm{Bo}$. However, the work done by gravity for perturbation energy is small for all Prandtl numbers.

\section{ACKNOWLEDGMENTS}

This work was supported by the National Natural Science Foundation of China (Grant Nos. 11872032, U1738119, and 11532015), the 2017 National Ministry of Science and Technology Additive Manufacturing Major Project (Grant No. 2017YFB1102900), and sponsored by the K. C. Wong Magna Fund at Ningbo University.

\section{APPENDIX: THE DERIVATION OF THE BASIC FLOW}

Substituting the form of basic flow (2.8) and the viscous stress tensor (2.5) into the momentum equation (2.3), we can derive that

$$
\begin{gathered}
x: 0=-\frac{\partial p}{\partial x}+\frac{\mathrm{d}^{2} u}{\mathrm{~d} z^{2}} \\
z: 0=-\frac{\partial p}{\partial z}+B o\left(-x+T_{b}(z)\right) .
\end{gathered}
$$


$p$ can be eliminated as follows:

$$
0=-\frac{\partial^{2} p}{\partial z \partial x}-B o, \quad 0=-\frac{\partial^{2} p}{\partial z \partial x}+-\frac{\mathrm{d}^{3} u}{\mathrm{~d} z^{3}}
$$

Therefore, we can obtain the velocity,

$$
u(z)=-\frac{B o}{6} z^{3}+C_{1} z^{2}+C_{2} z+C_{3}
$$

where $C_{1}=-\frac{3}{2} \zeta+\frac{3}{4}+\frac{5}{16} B o, C_{2}=3 \zeta-\frac{1}{2}-\frac{1}{8} B o$, and $C_{3}=-\zeta$. These constants are determined by the velocity on the plane $\left.u\right|_{z=0}=-\zeta$ from (2.6), the stress on the surface $\left.\tau_{13}\right|_{z=1}=\frac{\mathrm{d} u}{\mathrm{~d} z}=-\frac{\partial T}{\partial x}=1$ from (2.7), and the zero flux condition $\int_{0}^{1} u \mathrm{~d} z=0$. Then, substituting (A4) and (2.8) into (2.4), we can derive $-M a \cdot u=\frac{\mathrm{d}^{2}}{\mathrm{~d} z^{2}} T_{b}$, and so the temperature is

$$
T_{b}(z)=M a\left(\frac{B o}{120} z^{5}-\frac{C_{1}}{12} z^{4}-\frac{C_{2}}{6} z^{3}-\frac{C_{3}}{2} z^{2}\right)
$$

where the boundary condition $\left.\frac{\partial T}{\partial z}\right|_{z=1}=0$ in (2.7) and the assumption $\left.T_{b}\right|_{z=0}=0$ are used.

\section{REFERENCES}

${ }^{1}$ A. Karbalaei, R. Kumar, and H. J. Cho, "Thermocapillarity in microfluidics-A review," Micromachines 7, 13 (2016).

${ }^{2}$ R. B. R. Shankar Subramanian, The Motion of Bubbles and Drops in Reduced Gravity (Cambridge University Press, 2005).

${ }^{3}$ O. A. Basaran, H. J. Gao, and P. P. Bhat, "Nonstandard inkjets," Annu. Rev. Fluid Mech. 45, 85 (2013).

${ }^{4}$ H. H. Liu and Y. H. Zhang, "Modelling thermocapillary migration of a microfluidic droplet on a solid surface," J. Comput. Phys. 280, 37 (2015).

${ }^{5}$ V. Pratap, N. Moumen, and R. S. Subramanian, "Thermocapillary motion of a liquid drop on a horizontal solid surface," Langmuir 24, 5185 (2008).

${ }^{6} \mathrm{Z}$. B. Wu, "Steady thermocapillary migration of a droplet in a uniform temperature gradient combined with a radiation energy source at large Marangoni numbers," Phys. Rev. E 98, 013110 (2018).

${ }^{7}$ J. B. Bostwick, "Spreading and bistability of droplets on differentially heated substrates," J. Fluid Mech. 725, 566 (2013).

${ }^{8}$ M. K. Smith, "Thermocapillary migration of a 2-dimensional liquid droplet on a solid-surface," J. Fluid Mech. 294, 209 (1995).

${ }^{9}$ M. L. Ford and A. Nadim, "Thermocapillary migration of an attached drop on a solid surface," Phys. Fluids 6, 3183 (1994).

${ }^{10}$ Q. Dai, M. M. Khonsari, C. Shen, W. Huang, and X. Wang, "Thermocapillary migration of liquid droplets induced by a unidirectional thermal gradient," Langmuir 32, 7485 (2016).

${ }^{11}$ Q. W. Dai, W. Huang, X. L. Wang, and M. M. Khonsari, "Ringlike migration of a droplet propelled by an omnidirectional thermal gradient," Langmuir 34, 3806 (2018).

${ }^{12}$ T. Duffar, Crystal Growth Processes Based on Capillarity (John Wiley \& Sons, Ltd., USA, 2010).

${ }^{13}$ J. P. Singer, S. E. Kooi, and E. L. Thomas, "Focused laser-induced Marangoni dewetting for patterning polymer thin films," J. Polym. Sci., Part B: Polym. Phys. 54, 225 (2016).

${ }^{14}$ M. K. Smith and S. H. Davis, "Instabilities of dynamic thermocapillary liquid layers. Part I. Convective instabilities," J. Fluid Mech. 132, 119 (1983).

${ }^{15}$ R. J. Riley and G. P. Neitzel, "Instability of thermocapillary-buoyancy convection in shallow layers. Part 1 . Characterization of steady and oscillatory instabilities," J. Fluid Mech. 359, 143 (2000).
${ }^{16}$ Y. R. Li, N. Imaishi, T. Azami, and T. Hibiya, "Three-dimensional oscillatory flow in a thin annular pool of silicon melt," J. Cryst. Growth 260, 28 (2004).

${ }^{17}$ C. L. Chan and C. F. Chen, "Effect of gravity on the stability of thermocapillary convection in a horizontal fluid layer," J. Fluid Mech. 647, 91 (2010).

${ }^{18}$ L. Zhang, Y.-R. Li, C.-M. Wu, and L. Zhang, "Effect of surface heat dissipation on thermocapillary convection of moderate Prandtl number fluid in a shallow annular pool," J. Cryst. Growth 514, 21 (2019).

${ }^{19}$ L. A. Davalos-Orozco, "Sideband thermocapillary instability of a thin film flowing down the outside of a thick walled cylinder with finite thermal conductivity," Int. J. Non-Linear Mech. 109, 15 (2019).

${ }^{20}$ Q. Kang, J. Wang, L. Duan, Y. Su, J. He, D. Wu, and W. Hu, "The volume ratio effect on flow patterns and transition processes of thermocapillary convection," J. Fluid Mech. 868, 560 (2019).

${ }^{21} \mathrm{X}$. Zhong and F. Duan, "Stable hydrothermal waves at steady state evaporating droplet surface," Sci. Rep. 7, 16219 (2017).

${ }^{22}$ T.-S. Wang and W.-Y. Shi, "Influence of substrate temperature on Marangoni convection instabilities in a sessile droplet evaporating at constant contact line mode,” Int. J. Heat Mass Transfer 131, 1270 (2019).

${ }^{23}$ D. L. Albernaz, G. Amberg, and D.-Q. Minh, "Simulation of a suspended droplet under evaporation with Marangoni effects," Int. J. Heat Mass Transfer 97, 853 (2016).

${ }^{24}$ G. V. Kuznetsov, S. Y. Misyura, R. S. Volkov, and V. S. Morozov, "Marangoni flow and free convection during crystallization of a salt solution droplet," Colloids Surf., A 572, 37 (2019).

${ }^{25}$ S. Kim, J. Kim, and H.-Y. Kim, "Dewetting of liquid film via vapour-mediated Marangoni effect," J. Fluid Mech. 872, 100 (2019).

${ }^{26}$ G. Karapetsas, K. C. Sahu, and O. K. Matar, "Effect of contact line dynamics on the thermocapillary motion of a droplet on an inclined plate," Langmuir 29, 8892 (2013).

${ }^{27}$ L. Zheng, S. Zheng, and Q. Zhai, "Continuous surface force based lattice Boltzmann equation method for simulating thermocapillary flow,” Phys. Lett. A 380, 596 (2016).

${ }^{28}$ F. Brochard, "Motions of droplets on solid surfaces induced by chemical or thermal gradients," Langmuir 5, 432 (1989).

${ }^{29}$ M. K. Smith and S. H. Davis, "Instabilities of dynamic thermocapillary liquid layers. Part 2. Surface-wave instabilities,” J. Fluid Mech. 132, 145 (1983).

${ }^{30}$ K.-X. Hu, M. He, Q.-S. Chen, and R. Liu, "Effect of gravity on the stability of viscoelastic thermocapillary liquid layers," Int. J. Heat Mass Transfer 123, 776 (2018).

${ }^{31}$ M. Teitel, D. Schwabe, and A. Y. Gelfgat, "Experimental and computational study of flow instabilities in a model of Czochralski growth," J. Cryst. Growth 310, 1343 (2008).

${ }^{32}$ C.-Y. Yan, K.-X. Hu, and Q.-S. Chen, "Thermocapillary instabilities of liquid layers on an inclined plane," Phys. Fluids 30, 082101 (2018).

${ }^{33}$ H. K. Dhavaleswarapu, C. P. Migliaccio, S. V. Garimella, and J. Y. Murthy, "Experimental investigation of evaporation from low-contact-angle sessile droplets,” Langmuir 26, 880 (2010).

${ }^{34} \mathrm{~S}$. Takahashi and O. Kuboi, "Study on contact angles of $\mathrm{Au}, \mathrm{Ag}, \mathrm{Cu}, \mathrm{Sn}, \mathrm{Al}$ and Al alloys to SiC,” J. Mater. Sci. 31, 1797 (1996).

${ }^{35}$ P. J. Schmid and D. S. Henningson, Stability and Transition in Shear Flows (Springer-Verlag, 2001).

${ }^{36}$ M. K. Smith, "Instability mechanisms in dynamic thermocapillary liquid layers," Phys. Fluids 29, 3182 (1986).

${ }^{37}$ M. Wanschura, V. M. Shevtsova, H. C. Kuhlmann, and H. J. Rath, "Convective instability mechanisms in thermocapillary liquid bridges," Phys. Fluids 7, 912 (1995).

${ }^{38}$ K. X. Hu, J. Peng, and K. Q. Zhu, "The linear stability of plane Poiseuille flow of Burgers fluid at very low Reynolds numbers," J. Non-Newtonian Fluid Mech. 167, 87 (2012).

${ }^{39}$ A. Askounis, Y. Kita, M. Kohno, Y. Takata, V. Koutsos, and K. Sefiane, "Influence of local heating on Marangoni flows and evaporation kinetics of pure water drops," Langmuir 33, 5666 (2017). 
${ }^{40}$ S. Dash, A. Chandramohan, J. A. Weibel, and S. V. Garimella, "Buoyancyinduced on-the-spot mixing in droplets evaporating on nonwetting surfaces," Phys. Rev. E 90, 062407 (2014).

${ }^{41}$ J. B. Brzoska, F. Brochard-Wyart, and F. Rondelez, "Motions of droplets on hydrophobic model surfaces induced by thermal gradients," Langmuir 9, 2220 (1993).
${ }^{42}$ K. Sefiane, J. R. Moffat, O. K. Matar, and R. V. Craster, "Self-excited hydrothermal waves in evaporating sessile drops," Appl. Phys. Lett. 93, 074103 (2008).

${ }^{43}$ J. Z. Chen, S. M. Troian, A. A. Darhuber, and S. Wagner, "Effect of contact angle hysteresis on thermocapillary droplet actuation," J. Appl. Phys. 97, 014906 (2005). 